\section{Hemochromatosis proteins are dispensable for the acute hepcidin response to BMP2}

The liver iron hormone hepcidin regulates body iron homeostasis by blocking iron export via ferroportin. Hepcidin transcription is controlled by the BMP-SMAD pathway, whose activation requires BMP2, BMP6, type I (ALK2 and ALK3) and type II (ACVR2A and BMPR2) BMP receptors. ${ }^{1} \mathrm{BMP} 2$ and BMP6 are mainly expressed by liver sinusoidal endothelial cells and their deletion in mice causes iron overload due to decreased hepcidin mRNA expression. ${ }^{2,3}$ BMP type II receptors show a redundant function in hepcidin regulation in vivo, while ALK2 and ALK3 have a non-redundant role. The current model suggests that ALK2 is mainly involved in BMP6-dependent hepcidin upregulation in conditions of iron overload $^{4}$ and is inhibited by FKBP $12,{ }^{5}$ whereas ALK3 maintains basal hepcidin expression ${ }^{4}$ and signals preferentially in response to BMP2., ${ }^{6,7}$ Hereditary hemochromatosis $(\mathrm{HH})$, characterized by iron overload due to inappropriately low hepcidin production, is caused by mutations in

A

Hamp

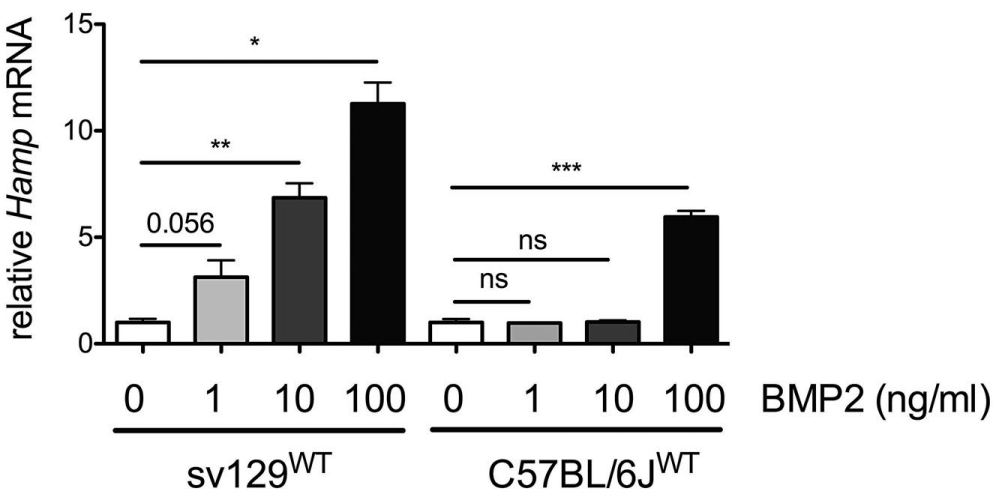

B
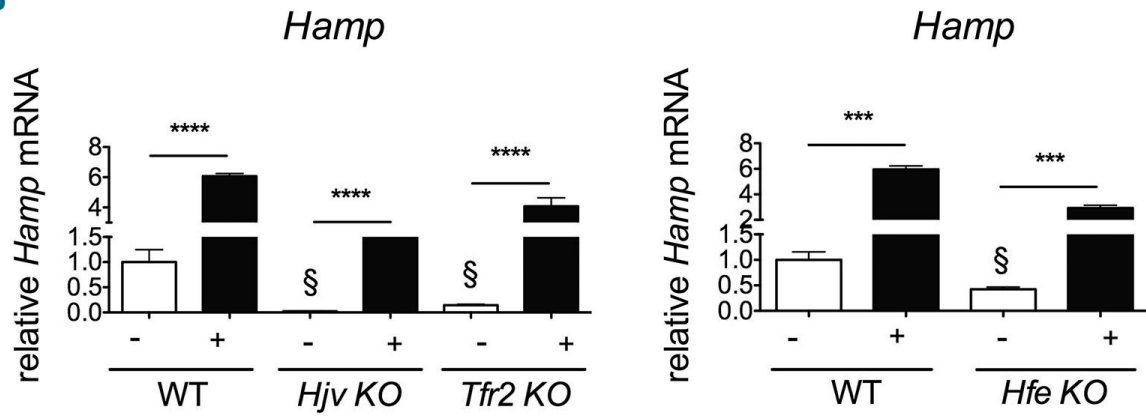

BMP2

C

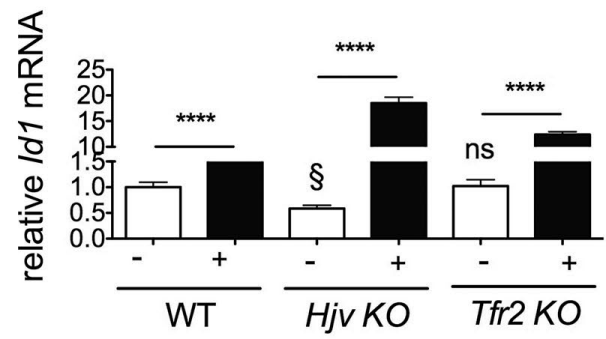

ld1

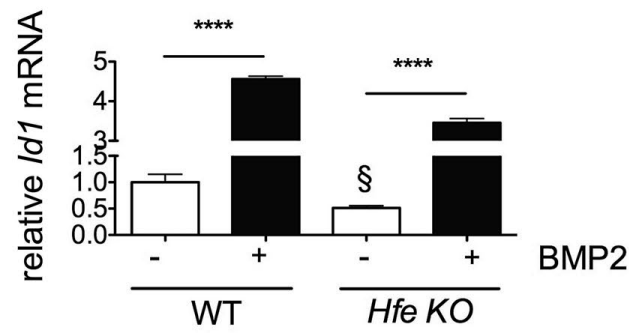

Figure 1. BMP2 up-regulates hepcidin and Id1 expression in murine primary hepatocytes lacking Hjv, Tfr2 or Hfe. (A) Primary murine hepatocytes from 3 or 4 wild-type (WT) male mice maintained on a Sv129 or C57BL/6J background, respectively, were isolated as described ${ }^{5}$ and treated for 4 hours (h) with increasing concentrations of BMP2 (1-100 ng/mL as indicated). Total RNA was isolated and retrotranscribed, and quantitative real-time polymerase chain reaction (qRTPCR) was performed to analyze hepcidin (Hamp) expression. Gapdh was used as housekeeping gene. mRNA expression ratio was normalized to an untreated mean value of 1 . A representative experiment is shown. (B and C) Primary murine hepatocytes were isolated from 3 WT, 3 Hjv-knockout (KO), and 3 Tfr2-KO mice (on a Sv129 background) and treated with $10 \mathrm{ng} / \mathrm{mL}$ BMP2 for $4 \mathrm{~h}$, or from 4 WT and 4 Hfe-KO mice (on a C57BL/6J background) and treated with 100 $\mathrm{ng} / \mathrm{mL}$ BMP2 for $4 \mathrm{~h}$. Total RNA was isolated and retrotranscribed, and qRT-PCR was performed to analyze hepcidin (Hamp) (B) and Id1 (C) expression. Gapdh was used as housekeeping genes. mRNA expression ratio was normalized to an untreated mean value of 1 . A representative experiment is shown. *Untreated versus BMP2-treated. suntreated WT versus untreated hereditary hemochromatosis. ${ }^{*} P<0.05 ; * * P<0.01 ; * * * P<0.001 ; * * * * P<0.0001 ;{ }^{s} P<0.05 ;$ ns: not significant. Error bar indicates standard error. 

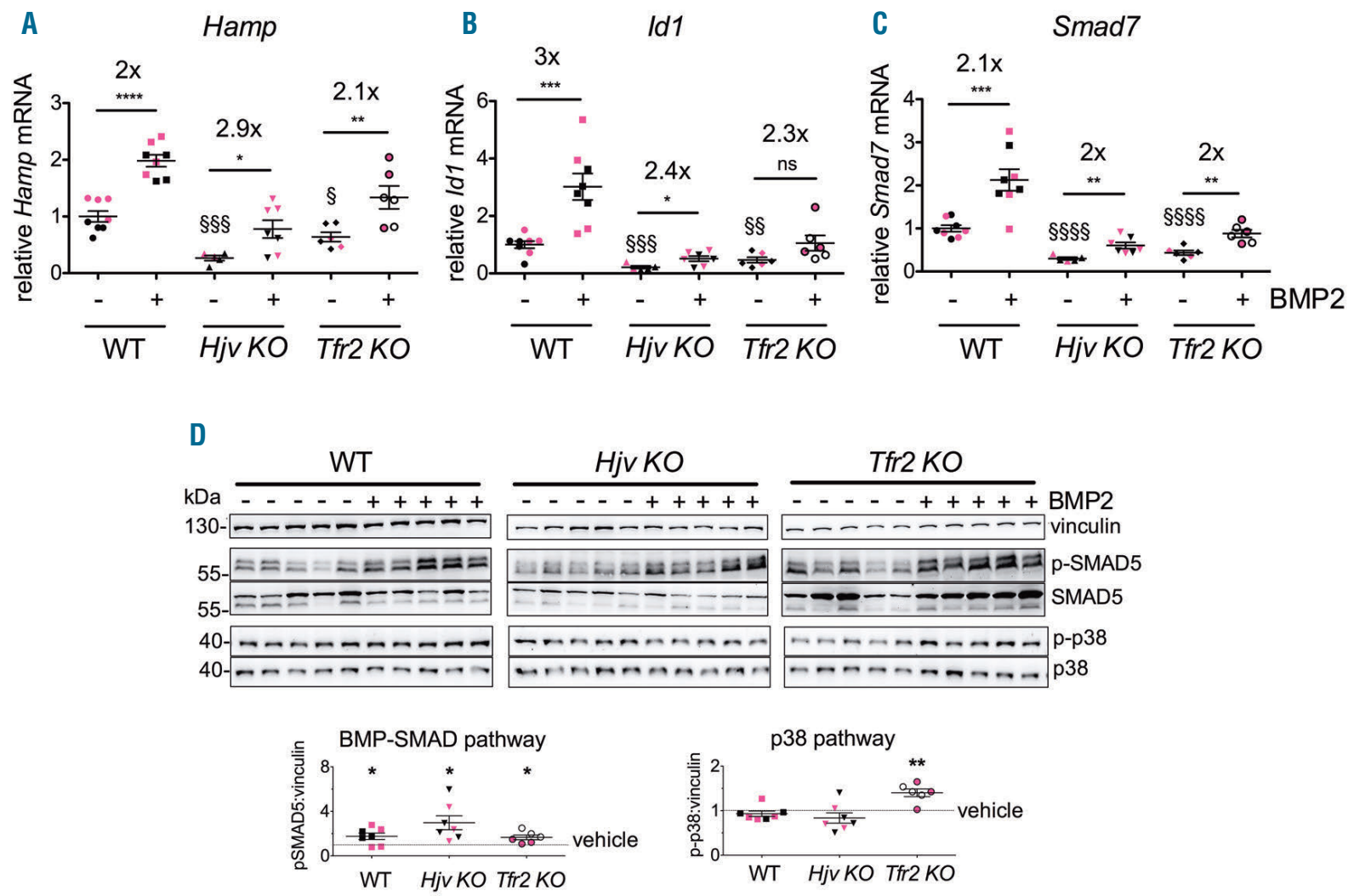

Figure 2. Acute BMP2 treatment in wild-type (WT), Hjv- and Tfr2-knockout (KO) mice up-regulates hepcidin through BMP-SMAD pathway activation. WT ( $=16$ ), Hjv-KO ( $=13)$, and Tfr2-KO ( $=12)$ mice (10-12 weeks old) were treated with vehicle or $24 \mu \mathrm{g} /$ mouse BMP2 and sacrificed 4 hours later. Total liver RNA was isolated and retrotranscribed, and quantitative real-time polymerase chain reaction was performed. Liver hepcidin (Hamp, A), Id1 (B) and Smad7 (C) mRNA levels were normalized to the housekeeping gene Hprt1. (D) Liver extracts were separated on a $12 \%$ SDS-PAGE for western blotting and probed with antibodies specific for phospho- or unmodified SMAD5 and p38. The anti-phospho-SMAD5 and anti-SMAD5 antibodies recognize two bands: according to Wang et al., ${ }^{16}$ SMAD5 corresponds to the lower band. Vinculin was used to normalize gel loading. Molecular weight markers are indicated on the left. (Right) Densitometric analyses of p-SMAD5 and p-p38 are indicated below. Males: black symbol; females: pink symbol. ${ }^{*} P<0.05 ; * * P<0.01 ; * * * P<0.001 ; * * * * P<0.0001$.

$H F E$, the second transferrin receptor (TFR2), hemojuvelin $(H J V)$ and hepcidin $(H A M P)$. HJV is a BMP co-receptor that activates the BMP-SMAD pathway and hepcidin expression. It interacts with HFE and TFR2 and is functionally independent of BMP6 and likely of ALK2. ${ }^{1}$ Recent studies imply a function of $\mathrm{HH}$ proteins in BMP2ALK3 signaling: both $\mathrm{HJV}^{8}$ and TFR2 ${ }^{9}$ interact with BMP2, and TFR2 and HFE bind ALK3 ${ }^{9,10}$ (data not shown). However, the functional role of the $\mathrm{HH}$ proteins in BMP2-mediated hepcidin regulation is still elusive.

To analyze whether HFE, TFR2 and HJV play a role in hepcidin activation by BMP2, we first studied primary murine hepatocytes (mHC) isolated from wild-type (WT) and $\mathrm{HH}$ mouse models. Since Hfe-KO mice were on the C57BL/6J and Tfr2 and Hjv-KO mice on the Sv129 genetic background, responses of Hjv, Hfe and Tfr2-KO primary $\mathrm{mHC}$ to $\mathrm{BMP} 2$ were compared to those of controls on the appropriate background. Unexpectedly, $\mathrm{mHC}$ isolated from C57BL/6J background mice showed a more than 10-fold diminished hepcidin response to BMP2 compared to Sv129 (Figure 1A). Thus the concentration of BMP2 used was $10 \mathrm{ng} / \mathrm{mL}$ for Sv129-derived cells and $100 \mathrm{ng} / \mathrm{mL}$ for C57BL/6J-derived hepatocytes. As expected, basal hepcidin expression was reduced in Hfe-, Tfr2and $H j v-\mathrm{KO} \mathrm{mHC}$. However, BMP2 treatment increased hepcidin expression independently of the genotype (Figure 1B and Online Supplementary Figure S1A), likely in a BMP-SMAD pathway-dependent manner as suggested by the upregulation of the BMP-SMAD target genes Id1 (Figure $1 \mathrm{C}$ and Online Supplementary Figure $S 1 B$ ). In the absence of $\mathrm{HH}$ proteins, the fold-increase of hepcidin and Id1 was more pronounced than in WT likely because of the low basal activation of the pathway or the high iron content of hepatocytes derived from $\mathrm{HH}$ models. These results indicate that $\mathrm{HH}$ proteins are dispensable for BMP2-dependent hepcidin upregulation ex vivo in hepatocytes.

To explore the in vivo role of $\mathrm{HH}$ proteins in BMP2dependent hepcidin activation, we focused on $H j v$ - and Tfr2-KO mice whose genetic background conferred increased $\mathrm{BMP} 2$ sensitivity. Adult $\mathrm{HH}$ mice and WT littermates were treated with a single BMP2 or saline injection and sacrificed 4 hours later. We chose the BMP2 dose (24 mg/mouse) that up-regulated both Hamp and Id 1 in WT mice (Online Supplementary Figure S2). Hjv-KO mice were severely iron loaded, with high liver iron (LIC) and serum iron levels (Online Supplementary Figures S3A and $C)$, and low spleen iron concentration (SIC) (Online Supplementary Figure $S 3 B$ ), whereas Tfr2-KO mice showed milder iron overload than $\mathrm{Hjv}$-KO mice (Online Supplementary Figure $S 3 A-C$ ). Iron parameters were not modified by the short BMP2 treatment in both models (Online Supplementary Figure $S 3 A-C$ ). The BMP-SMAD target genes hepcidin (Figure 2A and Online Supplementary Figure S3E), Id1 (Figure 2B and Online Supplementary Figure $S 3 F$ ), and Smad7 (Figure 2C and Online 
A

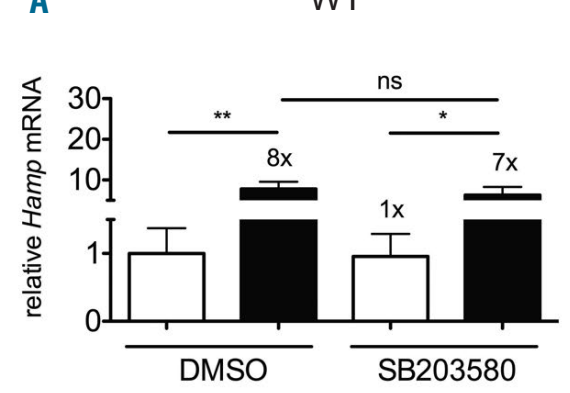

B

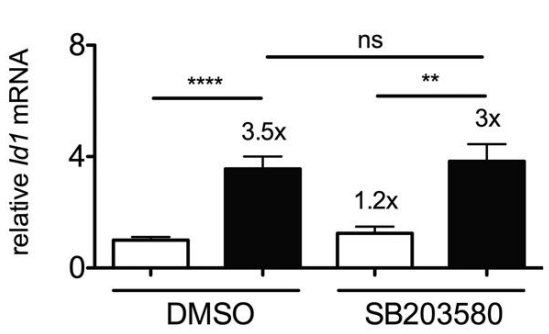

Hjv KO

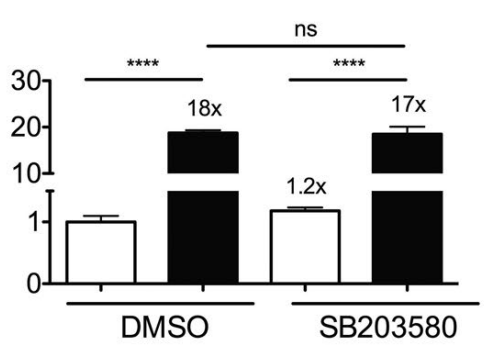

Hjv KO

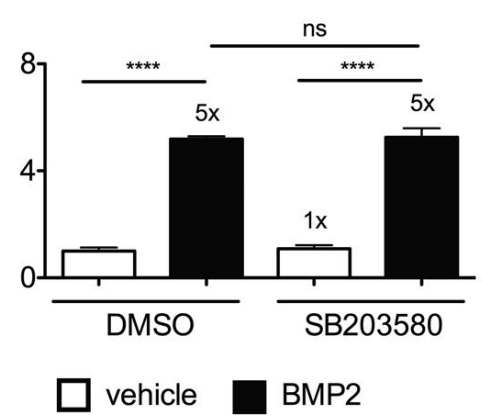

Tfr2 KO

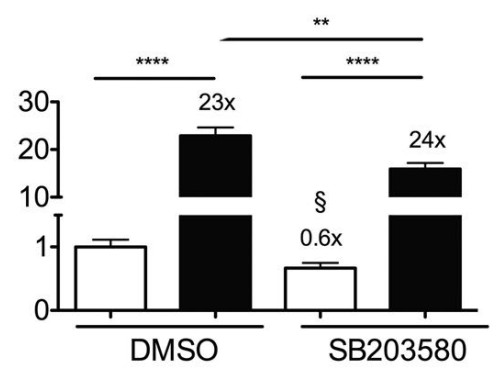

Figure 3. p38 activation contributes to BMP2-dependent hepcidin upregulation in Tfr2-knockout (KO) primary hepatocytes. (A and B) Primary hepatocytes were isolated from 3 wild-type (WT), 2 Hjv-KO and 2 Tfr2-KO (8-10 weeks old, females) and treated with BMP2 (10 ng/mL) in the presence or absence of the p38 inhibitor SB203580 (10 $\mathrm{MM})$. Expression of hepcidin (A) and Id1 (B) were assessed by quantitative real-time polymerase chain reaction. Gapdh was used as housekeeping gene. mRNA expression ratio was normalized to an untreated mean value of $1 . * P<0.05 ; * * P<0.01 ; * * * * P<0.0001 ;{ }^{\S} P<0.05 \mathrm{SB} 203580$ versus DMSO; ns: not significant. Error bar indicates standard error. Numbers over the white bars indicate fold changes of SB203580-treated cells versus DMSOtreated ones. Numbers over the black bars indicate fold changes of BMP2-treated cells versus vehicle-treated ones.

Supplementary Figure $S 3 G$ ) were significantly up-regulated in Hjv- and Tfr2-KO mice after BMP2 treatment. For our data analysis, we pooled male and female mice, since in our hands they showed comparable BMP-SMAD pathway activation and hepcidin expression. Accordingly, the analysis of male mice only showed that hepcidin (Online Supplementary Figure S4A) and Smad7 mRNA expression (Online Supplementary Figure $S 4 B$ ) were significantly increased by BMP2 both in WT and $\mathrm{HH}$ mice. Serum hepcidin mirrored Hamp mRNA in that it was increased in WT and $H j v-\mathrm{KO}$ mice and showed a trend towards increase in Tfr2-KO mice (Online Supplementary Figure $S 3 D)$. We exclude the possibility that the absence of HJV and TFR2 decreases BMP2 sensitivity; indeed the foldchange increase of hepcidin (Online Supplementary Figure $S 5 A$ ) and Id1 (Online Supplementary Figure S5B) was augmented in $\mathrm{HH}$-derived $\mathrm{HC}$.

Overall these results indicate that both HJV and TFR2 are dispensable for the transcriptional activation of hepcidin by BMP 2 in vivo, in agreement with results obtained in hepatocytes.

Bmp6 expression is strongly regulated by iron concentration. ${ }^{11}$ Consistently, Bmp6 mRNA levels were high in untreated $H j v$ - and Tfr2-KO mice compared to WT (Online Supplementary Figure S4C). By contrast, Bmp2 mRNA expression, which is only mildly responsive to iron, ${ }^{12}$ was comparable to WT mice and unaffected by iron overload in Hjv- and Tfr2-KO mice (Online Supplementary Figure $S 4 D$ ). Expression of both Bmp2 and Bmp6 was unchanged by BMP2 treatment.
BMP2 likely induces the activation of canonical (SMAD1/5/8) and of non-canonical signaling pathways, the latter culminating in AKT, ERK and p38 phosphorylation. ${ }^{13}$ We next investigated whether HJV and TFR2 influence the activation of these pathways in response to BMP2. Under basal conditions phospho-SMAD5 levels were strongly reduced in $H j v$-KO mice and similar to WT in Tfr2-KO mice, but still inappropriately low considering the increased LIC and Bmp6 (Online Supplementary Figure $S 6 A$ and $B$ ). AKT, ERK and p38 pathways were unaffected by the absence of HJV or TFR2 (Online Supplementary Figure $S 6 A, C-F)$.

Importantly, BMP2 injection increased SMAD5 phosphorylation both in WT and in HH mice (Figure 2D), further supporting the dispensable role of $\mathrm{HH}$ proteins for BMP-SMAD pathway activation in response to acute BMP2 treatment. Moreover, BMP2 fails to activate the non-canonical AKT and ERK pathways in all the genotypes (Online Supplementary Figure $S 7 A, C-E$ ), likely because of their fast kinetics. ${ }^{9}$ Surprisingly, BMP2 induces p38 phosphorylation only in Tfr2-KO mice (Figure 2D), a process that in turn might increase SMAD $1 / 5 / 8$ phosphorylation, as shown in other cell types. ${ }^{14}$ We speculate that in vivo Tfr2 deficiency enhances BMP2-mediated hepcidin expression through p38 phosphorylation. To investigate whether p38 phosphorylation contributes to hepcidin upregulation, WT, $H j v-\mathrm{KO}$ and $T f r 2-\mathrm{KO}$ primary $\mathrm{HC}$ were treated with $\mathrm{BMP} 2$ in the presence or absence of the p38 inhibitor SB203580. SB203580 treatment in primary HC up-regulated Egr1 expression (Online 
Supplementary Figure S8), as described, ${ }^{15}$ confirming p38 inhibition. It did not change basal and BMP2-mediated hepcidin upregulation in WT and $H j v-\mathrm{KO} \mathrm{HC}$. Interestingly, SB203580 significantly reduced hepcidin expression both in untreated and in BMP2-treated Tfr2-KO HC (Figure 3A) in a SMAD1/5/8-independent way, as shown by comparable Id1 expression (Figure $3 \mathrm{~B}$ ). However, the change increase by BMP2 is comparable in the untreated and SB203580-treated cells. Overall these results suggest a functional role of TFR2 in p38 regulation. Whether this signaling pathway influences hepcidin regulation still remains to be clarified.

In summary, our results demonstrate that HJV and TFR2 are necessary for the canonical BMP-SMAD pathway but not for the non-canonical pathways in steady state, and that $\mathrm{HH}$ proteins are not required for the activation of $\mathrm{BMP}-\mathrm{SMAD}$ signaling and hepcidin expression by BMP2.

Our study highlights the dispensable role of the $\mathrm{HH}$ proteins in response to an acute BMP2 increase, likely occurring in response to increased iron levels, ${ }^{12}$ and uncovers an unprecedented role of TFR2 in the regulation of p38 signaling.

\section{Alessia Pagani, ${ }^{1 *}$ Mariateresa Pettinato, ${ }^{1,2 *}$ Silvia Colucci, ${ }^{3 *}$ Alessandro Dulja, ${ }^{20}$ Martina Rauner, ${ }^{4,5}$ Antonella Nai, ${ }^{1,2}$ Clara Camaschella, ${ }^{1}$ Sandro Altamura, ${ }^{3 \#}$ Martina U. Muckenthaler ${ }^{3 \#}$ and Laura Silvestri ${ }^{1,2 \#}$}

'Division of Genetics and Cell Biology, San Raffaele Scientific Institute, Milan, Italy; ${ }^{2}$ Vita-Salute San Raffaele University, Milan, Italy; ${ }^{3}$ Department of Pediatric Hematology, Oncology and Immunology, University of Heidelberg, and MMPU-Molecular Medicine Partnership Unit, Heidelberg, Germany; ${ }^{4}$ Department of Medicine III, Technische Universität Dresden, Dresden, Germany and ${ }^{5}$ Center for Healthy Aging, Technische Universität Dresden, Dresden, Germany

*AP, MP and SC contributed equally as co-first authors.

"SA, MUM and LS contributed equally as co-senior authors.

${ }^{\circ}$ Current address: Genome Biology Unit, European Molecular Biology

Laboratory (EMBL), Heidelberg, Germany

Correspondence: LAURA SILVESTRI - silvestrilaura@hst.it MARTINA U. MUCKENTHALER martina.muckenthaler@med.uni-heidelbero.de

doi:10.3324/haematol.2019.241984
Funding: the work was supported by EHA-Advanced Research Grant to AP and SA. MUM acknowledges funding from the Deutsche Forschungsgemeinschaft (SFB1036).

\section{References}

1. Silvestri L, Nai A, Dulja A, Pagani A. Hepcidin and the BMP-SMAD pathway: An unexpected liaison. Vitam Horm. 2019;110:71-99.

2. Koch PS, Olsavszky V, Ulbrich F, et al. Angiocrine Bmp2 signaling in murine liver controls normal iron homeostasis. Blood. 2017; 129(4):415-419.

3. Canali S, Zumbrennen-Bullough $\mathrm{KB}$, Core $\mathrm{AB}$, et al. Endothelial cells produce bone morphogenetic protein 6 required for iron homeostasis in mice. Blood. 2017;129(4):405-414.

4. Steinbicker AU, Bartnikas TB, Lohmeyer LK, et al. Perturbation of hepcidin expression by BMP type I receptor deletion induces iron overload in mice. Blood. 2011;118(15):4224-4230.

5. Colucci S, Pagani A, Pettinato M, et al. The immunophilin FKBP12 inhibits hepcidin expression by binding the BMP type I receptor ALK2 in hepatocytes. Blood. 2017;130(19):2111-2120.

6. Xia Y, Babitt JL, Sidis Y, Chung RT, Lin HY. Hemojuvelin regulates hepcidin expression via a selective subset of BMP ligands and receptors independently of neogenin. Blood. 2008;111(10):5195-5204

7. Wang CY, Xu Y, Traeger L, et al. Erythroferrone lowers hepcidin by sequestering BMP2/6 heterodimer from binding to the BMP type I receptor AL K3. Blood. 2020:135(6):453-456.

8. Healey EG, Bishop B, Elegheert J, Bell CH, Padilla-Parra S, Siebold C Repulsive guidance molecule is a structural bridge between neogenin and bone morphogenetic protein. Nat Struct Mol Biol. 2015; 22(6):458-465

9. Rauner M, Baschant $U$, Roetto A, et al. Transferrin receptor 2 controls bone mass and pathological bone formation via BMP and Wnt signaling. Nat Metab. 2019;1(1):111-124.

10. Wu XG, Wang Y, Wu Q, et al. HFE interacts with the BMP type I receptor ALK3 to regulate hepcidin expression. Blood. 2014; 124(8):1335-1343

11. Kautz L, Meynard D, Monnier A, et al. Iron regulates phosphorylation of Smad1/5/8 and gene expression of Bmp6, Smad7, Id1, and Atoh8 in the mouse liver. Blood. 2008:112(4):1503-1509.

12. Canali S, Wang CY, Zumbrennen-Bullough KB, Bayer A, Babitt JL. Bone morphogenetic protein 2 controls iron homeostasis in mice independent of Bmp6. Am J Hematol. 2017;92(11):1204-1213.

13. Wang RN, Green J, Wang Z, et al. Bone Morphogenetic Protein (BMP) signaling in development and human diseases. Genes Dis. 2014:1(1):87-105

14. Yang G, Yuan G, Li X, Liu P, Chen Z, Fan M. BMP-2 induction of Dlx3 expression is mediated by $\mathrm{p} 38 / \mathrm{Smad} 5$ signaling pathway in osteoblastic MC3T3-E1 cells. J Cell Physiol. 2014;229(7):943-954.

15. Whitmarsh AJ. A central role for p38 MAPK in the early transcriptional response to stress. BMC Biol. 2010:8:47.

16. Wang $C-Y$, Core $A B$, Canali $S$, et al. Smad1/5 is required for erythropoietin-mediated suppression of hepcidin in mice. Blood 2017;130 (1):73-83. 